\title{
Protective mechanism of quercetin on acute myocardial infarction in rats
}

\author{
B. Li ${ }^{1}$, M. Yang ${ }^{2}$, J.W. Liu ${ }^{1}$ and G.T. Yin ${ }^{1}$ \\ ${ }^{1}$ Department of Cardiology II, \\ The Third Affiliated Hospital of Xinxiang Medical University, Xinxiang, China \\ ${ }^{2}$ Department of Cardiology I, \\ The Third Affiliated Hospital of Xinxiang Medical University, Xinxiang, China \\ Corresponding author: G.T. Yin \\ E-mail: guotianyincn@163.com \\ Genet. Mol. Res. 15 (1): gmr.15017117 \\ Received September 30, 2015 \\ Accepted November 30, 2015 \\ Published March 11, 2016 \\ DOI http://dx.doi.org/10.4238/gmr.15017117
}

ABSTRACT. To investigate the protective mechanism of quercetin on acute myocardial infarction (AMI) rats, an AMI rat model was established by ligating the left coronary anterior descending branch. The rats were randomly divided into the model group and low- and high-dose quercetin groups. The control group comprised sham-operated rats. The rats in the low- and high-dose quercetin groups were administered 100 and $400 \mathrm{mg} /$ $\mathrm{kg}$ quercetin, respectively, by gavage. The rats in the control and model groups were administered isometric normal saline once daily for one week. The mRNA and protein levels of TNF- $\alpha$ and IL-1 $\beta$ in the myocardial tissue of rats were detected in each group by real time polymerase chain reaction and enzyme-linked immunosorbent assay. Malondialdehyde (MDA) content in the myocardial tissue and superoxide dismutase (SOD) and catalase (CAT) activities were detected using a colorimetric method. The level of apoptosis was detected by terminal deoxynucleotidyl transferase dUTP nick end labeling. Compared with those in the control group, the mRNA and protein levels of TNF- $\alpha, \mathrm{IL}-1 \beta$ and MDA content in the model, low-, and high-dose groups significantly increased. SOD and CAT activities decreased significantly. The cell apoptosis index increased significantly 
$(P<0.05)$. Compared with those in the model group, the mRNA and protein levels of TNF- $\alpha$ and IL- $1 \beta$ and MDA content in myocardial tissue of rats in the low-dose and high-dose groups decreased significantly. SOD and CAT activities increased significantly. The cell apoptosis index significantly reduced $(P<0.05)$. In conclusion, quercetin has significant anti-inflammatory, antioxidant, and anti-apoptotic effects on AMI rats and can effectively protect against myocardium damage.

Key words: Quercetin; Acute myocardial infarction; Cell apoptosis

\section{INTRODUCTION}

Coronary heart disease is one of the most common cardiovascular diseases threatening human health. Acute myocardial infarction (AMI), the basis of coronary artery disease, refers to a sudden blockage of the coronary artery followed by a sharp reduction in blood supply, severe and persistent acute myocardial ischemia, and ultimately myocardial necrosis. AMI is the primary cause of malignant arrhythmia, cardiac insufficiency, and sudden cardiac death (Devaux et al., 2012; Rahim et al., 2015). The onset of AMI is acute and the disease has a high incidence, rapid progression, and high mortality. At present, there are 17,000,000 people who die of cardiovascular diseases worldwide annually. More than half die of AMI. The incidence of AMI is significantly higher in developed countries. The incidence of AMI is increasing in China because of the aging population, eating habits, and the fast-pace society. In the past 10 years, the incidence of AMI patients has increased significantly in China and is now close to the international average level (Su et al., 2012). Recent research shows that oxidative stress is the main factor promoting the development of AMI. The generation and aggregation of a large number of oxygen free radicals causes cell structure and function damage. The inflammatory factors also increase the apoptosis of myocardial cells. Myocardial cell structure and function disorder can be induced through activation of cytotoxic T cells (Misra et al., 2009; Yang et al., 2014). Quercetin is a kind of flavonoid compound with biological activities. Abundant research in the past 10 years has confirmed the anti-tumor, anti-inflammatory, anti-platelet aggregation, anti-oxygen free radicals, and pro-vascular dilation properties of quercetin (Yao et al., 2011; Costa et al., 2013). In this study, the effects of quercetin on inflammatory factors, cell apoptosis, and oxygen free radicals in the myocardial tissue of AMI rats were investigated. The anti-inflammatory and antioxidant myocardial protective mechanisms were explored.

\section{MATERIAL AND METHODS}

\section{Establishment and treatment of AMI rat model}

Healthy Sprague Dawley rats (SPF, 200-220 g) were purchased from the Experimental Animal Center of Xinxiang Medical University, Xinxiang, China. This study was performed in strict accordance with the recommendations in the Guide for the Care and Use of Laboratory Animals of the National Institutes of Health Eighth Edition, 2010 (Bethesda, MD, USA). The animal use protocol was reviewed and approved by the Institutional Animal Care and Use Committee of The Third Affiliated Hospital of Xinxiang Medical University. Chloral hydrate (10\%) was used to anesthetize 45 rats by intraperitoneal injection at a dose of $0.3 \mathrm{~mL} / 100 \mathrm{~g}$. A 24-G trochar tube used as 
an endotracheal tube was inserted into the trachea. The skin was cut open from the left fourth intercostal space. The muscles were bluntly separated with forceps. The thoracic cavity was opened. The pericardium was clamped and damaged. The heart was extruded. The heart wall was pierced with 6.0 non-invasive thread along the coronary left anterior descending branch and ligated (if the myocardial color became shallow ligation was deemed successful). Then, the heart was quickly placed into the thoracic cavity. The thoracic cavity was sutured with a No. 0 suture. lodine disinfection was performed after the operation. Penicillin $(400,000 \mathrm{U} / \mathrm{kg})$ was intramuscularly injected into the rat hind limb to prevent infection. The tracheal catheter was pulled out after the rats recovered spontaneous breathing. Rat models (45 total) were successfully established and randomly divided into three equal groups: the model, low-dose, and high-dose quercetin groups. Another 15 rats were placed in the sham operation group (also called the control group, coronary artery ligation was not performed). The rats in the low- and high-dose groups were administered 100 and 400 $\mathrm{mg} / \mathrm{kg}$ quercetin, respectively (Yuanye Biotechnology Co., Shanghai, China) by gavage. The rats in the control group and model group were administered isometric normal saline in the postoperative $24 \mathrm{~h}$ once a day for 1 week. The rats were executed after treatment. The hearts were quickly removed and rinsed with physiological saline. The adipose tissue and other non-heart tissues were removed. The myocardial segment with maximum ventricular circumference was collected.

\section{RT-PCR}

Myocardial tissue $(0.1 \mathrm{~g})$ was placed in a glass homogenizer with $1 \mathrm{~mL}$ Trizol (Invitrogen, Carlsbad, CA, USA). The tissue was prepared in an ice bath and was homogenized until the homogenate was transparent without particles. The homogenate was transferred to a 1.5-mL EP tube. The supernatant was transferred to a new EP tube after centrifugation. Chloroform $(200 \mu \mathrm{L})$ was added and the mixture was vortexed. The mixture was left standing for $5 \mathrm{~min}$ and then centrifuged for $15 \mathrm{~min}$ at $12,000 \mathrm{rpm}$. The supernatant was transferred to a new EP tube and isometric isopropyl alcohol was added and mixed. It was left standing for $15 \mathrm{~min}$ and then centrifuged for $10 \mathrm{~min}$ at $12,000 \mathrm{rpm}$. The supernatant was discarded. Precooled $75 \%$ ethanol was added to wash and precipitate twice. RNase-free water was then added to dissolve the precipitate. The sample concentration was detected. RNA was transcribed into cDNA using a reverse transcription kit (TaKaRa, Dalian, China) and was used as the PCR template. The following primers were designed according to TNF- $\alpha$ and $L-1 \beta$ mRNA sequences provided by GeneBank. The related primers were as follows: TNF- $\alpha-F:$ 5'-GGGTGTTCATCCATTCTC-3', TNF- $\alpha-R:$ 5'-CCCAGCATCTTGTGTTTC-3'; IL-1 $\beta-F$ :

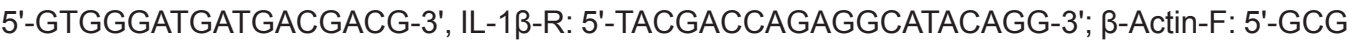
GGAAATCGTGCGTGAC-3', $\beta$-Actin-R: 5'-CGTCATACTCCTGCTTGCTG-3'. Sterilized distilled water $(5 \mu \mathrm{L}), 12.5 \mu \mathrm{L}$ SYBR Green general type qPCR Master Mix (Roche, Basel, Switzerland), $1 \mu \mathrm{L}$ various upstream/downstream primers $(10 \mu \mathrm{M})$, and $2 \mu \mathrm{L}$ cDNA template were added to the PCR tube. The total reaction volume was $25 \mu \mathrm{L}$. The reaction conditions were pre-degeneration: $94^{\circ} \mathrm{C}$ for $30 \mathrm{~s}$; degeneration: $94^{\circ} \mathrm{C}$ for $5 \mathrm{~s}$; annealing: $94^{\circ} \mathrm{C}$ for $30 \mathrm{~s}$; extension: $72^{\circ} \mathrm{C}$ for $40 \mathrm{~s}$; 35 cycles, and terminal extension for $5 \mathrm{~min}$ at $72^{\circ} \mathrm{C}$. The reaction was terminated. The dissolution curve was constructed. The data were directly read from the ABI 7500 real-time PCR instrument (Applied Biosystems, Foster City, CA, USA).

\section{ELISA}

The myocardial tissue $(0.5 \mathrm{~g})$ of rats from all groups was weighed and placed in a small 
beaker. Precooled saline was added at a volume approximately 9 times the tissue. The tissue block was rapidly cut into pieces with ophthalmic scissors and $200 \mu \mathrm{L}$ tissue lysate was added. The tissue was homogenized for $5 \mathrm{~min}$ with a homogenizer, placed on ice for $20 \mathrm{~min}$, and centrifuged for $15 \mathrm{~min}$ at $12,000 \mathrm{rpm}$. The supernatant was carefully extracted. The total protein concentration was detected with a BCA protein assay kit (Jingmei Biotechnology Co., Nanjing, China). ELISA was performed according to the manufacturer protocol (Jingmei Biotechnology Co., Nanjing, China). The concentrations of TNF- $\alpha$ and IL- $1 \beta$ were detected. Samples and standards were assayed in triplicate. The OD value was measured at $492 \mathrm{~nm}$ and the averages were calculated.

\section{Colorimetric determination}

The rat myocardial tissue samples were used for pretreatment. The supernatant was used for the malondialdehyde (MDA) assay (Meilian Biotechnology Co., Shanghai, China), superoxide dismutase (SOD) activity detection (Amyjet Scientific Inc., Wuhan, China), and catalase (CAT) activity detection (Amyjet Scientific Inc., Wuhan, China). OD values were measured at 532, 550, and $450 \mathrm{~nm}$ respectively. The concentration of standard was taken as the abscissa and OD values taken as the ordinate. The standard curve was drawn. The contents of MDA and activities of SOD and CAT in the rat myocardial tissue were calculated.

\section{Terminal deoxynucleotidyl transferase dUTP nick end labeling (TUNEL) assay}

The fresh myocardial tissue was embedded in paraffin, immersed twice in xylene for 5 min, immersed in ethanol $(100,95,90,80$, and $70 \%) 3$ min each time, incubated for 15 min with Proteinase $\mathrm{K}$ working solution, rinsed twice with PBS for 3 min each time, and then the TUNEL reaction mixture (Roche, Basel, Switzerland) was prepared. After the slides were dried, $50 \mu \mathrm{LU}$ NEL reaction mixture was added to the sample (only $50 \mu \mathrm{L}$ fluorescein labeled dUTP was added in the negative control group). The slides were kept in a humidity chamber for $1 \mathrm{~h}$ at $37^{\circ} \mathrm{C}$. PBS was used to rinse the slides 3 times for 3 min each time. One drop of PBS was added to count apoptotic cells under a fluorescence microscope and to take photos. The positive nuclei/total nuclei under 5 different high magnifications were counted on each section. The mean value was taken as the myocardial cell apoptosis index (Al).

\section{Statistical analysis}

All data were analyzed using the SPSS 17.0 statistical software (SPSS Inc., Chicago, IL, USA). The measurement data are reported as means \pm standard deviation. The comparison between measurement data of all groups was determined using analysis of variance. A side-by-side comparison between groups was determined using the LSD method. $\mathrm{P}<0.05$ was considered statistically significant.

\section{RESULTS}

\section{Comparison between TNF- $\alpha$ and IL-1 $\beta$ mRNA levels in rat myocardial cells}

As shown in Figure 1, compared with that in the control group, TNF- $\alpha$ and IL-1 $\beta$ mRNA levels in the model, low-dose, and high-dose groups increased significantly $(P<0.05)$. Compared 
with that in the model group, TNF- $\alpha$ and IL-1 $\beta$ mRNA levels in the low-dose group and high-dose group decreased significantly $(P<0.05)$. The TNF- $\alpha$ and IL-1 $\beta$ mRNA levels in the high-dose group were lower than those of the low-dose group $(P<0.05)$.
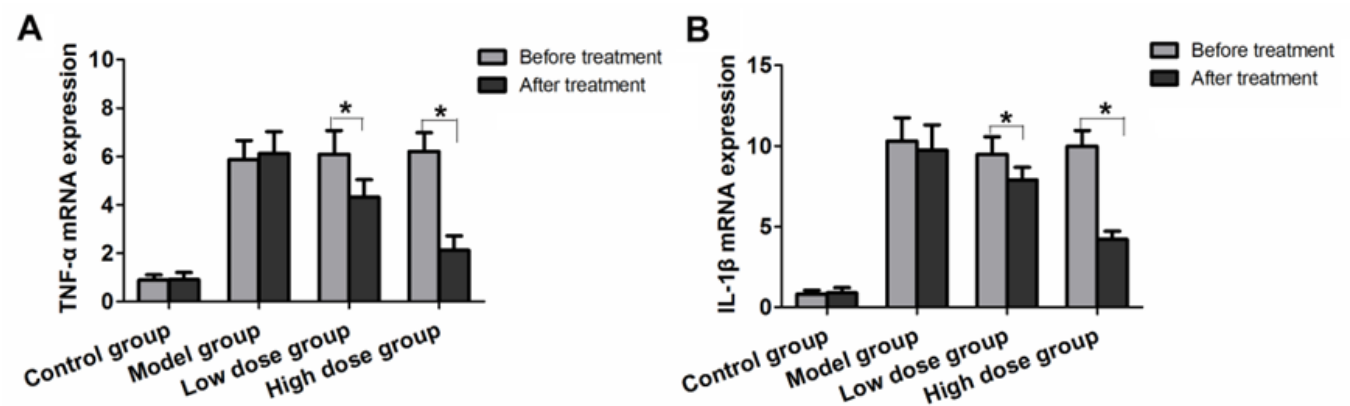

Figure 1. Comparison of TNF- $\alpha$ and IL-1 $1 \beta$ mRNA levels in rat myocardial cells. A. TNF- $\alpha$ mRNA in each group. B. IL-1 $\beta$ mRNA in each group. ${ }^{*} \mathrm{P}<0.05$.

\section{Comparison between TNF- $\alpha$ and IL-1 $\beta$ protein levels in rat myocardial cells}

Compared with that in the control group, TNF- $\alpha$ and IL-1 $\beta$ protein secretion in the model, low-dose, and high-dose groups increased significantly $(P<0.05)$ (Figure 2). Compared with that in the model group, TNF- $\alpha$ and IL-1 $\beta$ protein secretion in the high- and low-dose groups decreased significantly $(P<0.05)$. TNF- $\alpha$ and IL-1 $\beta$ protein levels in the high-dose quercetin group were lower than those of the low-dose group $(P<0.05)$ (Figure 2).
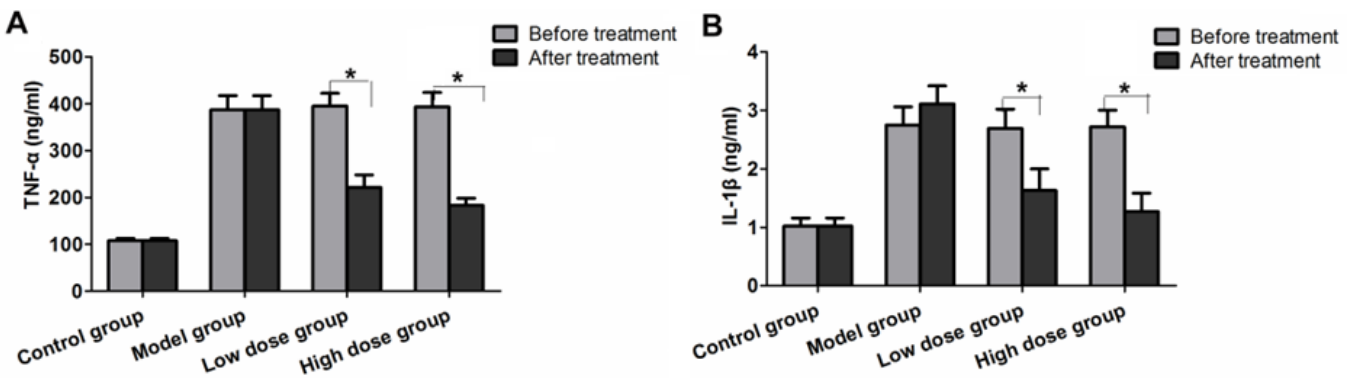

Figure 2. Comparison of TNF- $\alpha$ and IL-1 $\beta$ protein levels in rat myocardial cells. TNF- $\alpha$ and IL-1 $\beta$ protein levels in control, model, low-dose, and high-dose groups. ${ }^{*} \mathrm{P}<0.05$.

\section{Comparison between MDA content and SOD and CAT activities in the myocardial tissue}

As shown in Figure 3, compared with the control group, MDA content in the model, lowdose, and high-dose groups increased significantly $(P<0.05)$. The SOD and CAT activity decreased significantly $(P<0.05)$. Compared with that in the model group, the contents of MDA in the high- and low-dose groups decreased significantly $(P<0.05)$. The SOD and CAT activity increased significantly $(P<0.05)$. The change in the various indicators in the high-dose group was more significant than that in the low-dose group $(P<0.05)$ (Figure 3$)$. 
A

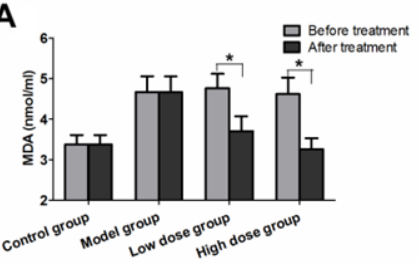

B

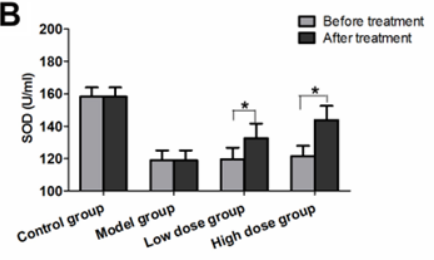

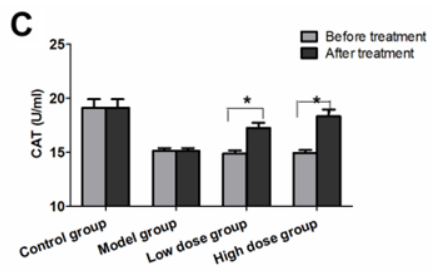

Figure 3. Comparison of MDA content and SOD and CAT activities in the myocardial tissue of rats. A. Comparison of MDA content of each group. B. Comparison of SOD activity of each group. C. Comparison of CAT activity of each group. ${ }^{*} \mathrm{P}<0.05$.

\section{Comparison of myocardial $\mathrm{Al}$ in rats}

The TUNEL detection results are shown in Figure 4. Compared with that in the control group, myocardial cell apoptosis was significantly higher in the model, low-dose, and high-dose groups $(P<0.05)$. Compared with that in the model group, the myocardial cell apoptosis in the high-dose and low-dose groups decreased significantly $(P<0.05)$, and the decrease in the highdose group was more significant than that in the low-dose group $(P<0.05)$.

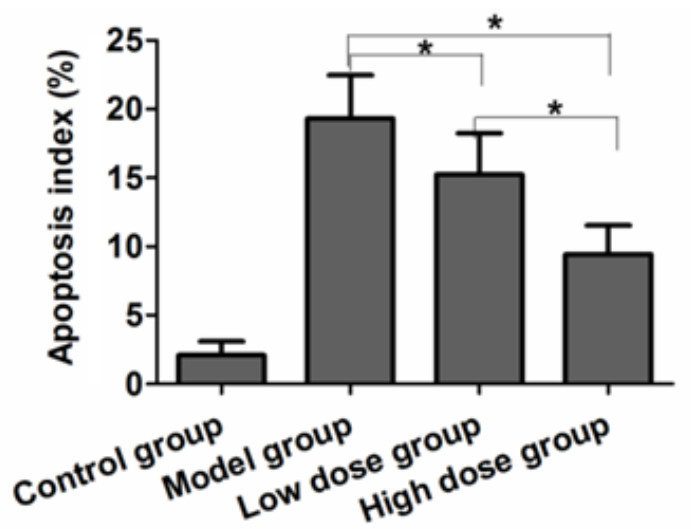

Figure 4. Comparison of myocardial apoptosis index in rats. ${ }^{*} \mathrm{P}<0.05$.

\section{DISCUSSION}

AMI refers to acute myocardial ischemic necrosis. The blood supply to the coronary artery is drastically reduced or interrupted. The corresponding myocardium experiences serious and persistent acute ischemia, resulting in myocardial necrosis. Arrhythmia often occurs in the early stages of AMI. Ventricular arrhythmia is a common fatal arrhythmia. Its mechanism is still not clear (Jeong et al., 2013; Jutla et al., 2014). It is believed that its pathogenesis involves plaque rupture and hemorrhage, thrombosis formation, and a sharp decrease in blood supply, which causes acute and persistent ischemia and hypoxia of the corresponding myocardium (Kivrak et al., 2014). The AMI incidence rate indicates a substantial annual growth in China and subsequently the mortality is increasing. The statistical results of 161 monitoring points in a national disease surveillance system utilized by Zhang et al. (2012) showed that the crude mortality of AMI rose from 40.4/100,000 to $45.7 / 100,000$ in 2008. 
The blood supply of various tissues and organs is decreased after AMI, resulting in a metabolism and internal environment imbalance, a large number of free radicals, and an activated mononuclear phagocyte system. Oxidative stress is a main factor contributing to AMI (He et al., 2012). Damage from oxygen free radicals induces peroxidation of many macromolecules in the cell. Excess oxygen free radicals directly damage myocardial cell organelles and membranes, especially the mitochondria, resulting in an increase in membrane permeability. MDA is the end product of unsaturated fatty acid oxidative metabolism and its content indirectly reflects the degree of oxidative damage. SOD and CAT are important free radical scavengers in vivo. Their catalytic abilities are important for maintaining oxidation balance. AMI induces changes in signaling pathways, resulting in a decrease in SOD and CAT activity (Andreadou et al., 2009; Zhou et al., 2011; Chahine et al., 2014). Zhang et al. (2013) showed that the content of MDA in serum and myocardial tissue of rats increased significantly through the establishment of the AMI rat model, and eNOS and ISOD activities significantly decreased, thus confirming oxidative damage to the myocardial tissue. The study showed that AMI could also cause tissue necrosis and inflammatory cell infiltration. Myocardial tissue secreted a large amount of TNF- $\alpha$, IL-1 $\beta$, and other inflammatory factors, and the reaction was amplified systematically. The inappropriate accumulation of abnormally expressed TNF- $\alpha$, IL-1 $\beta$, and other inflammatory factors could cause structure and function disorder of myocardial cells, inflammation response syndrome, and aggravation of myocardial tissue hypoxia through the activation of cytotoxic T cells (Kong et al., 2011). Toldo et al. (2013) investigated the effect of IL-1 $\beta$ on mice hearts through the establishment of an AMI mouse model. The results showed that IL-1 $\beta$ mediated an aseptic inflammatory reaction process having harmful effects. In this study, the mRNA and protein levels of TNF- $\alpha$ and IL-1 $\beta$ and MDA content in the myocardial tissue significantly increased, and SOD and CAT activities significantly decreased. The cell Al also significantly increased, which was consistent with previous research results.

Quercetin is a flavonoid compound. In vitro studies have shown that it has vast pharmacological functions and biological activities such as antioxidant properties, ability to scavenge oxygen free radicals, promote anti-platelet aggregation, lower blood pressure, protect the myocardium against ischemia, prevent ischemic reperfusion injury, enhance immune function, anticancer properties, and antibiosis, anti-virus, and analgesic effects (Youn et al., 2013). Therefore, quercetin is widely used in the treatment of ovarian cancer, breast cancer, white blood cell regulation, gastrointestinal tumors, coronary heart disease, hypertension, and other diseases. The powerful antioxidant quercetin could be used to reduce ischemia reperfusion injury of various organs including the liver, kidney, heart, and brain and improve cell function. An in vitro study confirmed that quercetin also has the ability to inhibit TNF- $\alpha$, IL-1 $\beta$, and other factors overproduced by the induction of LPS. Free radicals damage protein and nucleic acids during the oxidative stress response. Quercetin can scavenge free radicals and downregulate the downstream signaling pathway mediated by reactive oxygen species, thereby regulating intracellular signal molecules and protecting cells from oxidative stress injury (Lee et al., 2013).

This study investigated the protective effect of quercetin in an AMI rat model. The results showed that, compared with that in the model group, the mRNA and protein levels of TNF- $\alpha$ and IL-1 $\beta$ and MDA content significantly decreased. SOD and CAT activity significantly increased, and the cell Al decreased significantly. This indicates that quercetin had a significant anti-inflammatory, antioxidant, and anti-apoptotic effect on the myocardium of AMI rats and could effectively protect against myocardium injury. The above results provide a theoretical foundation for the clinical treatment of AMl using quercetin. 


\section{REFERENCES}

Andreadou I, lliodromitis EK, Farmakis D and Kremastinos DT (2009). To prevent, protect and save the ischemic heart: antioxidants revisited. Expert Opin. Ther. Targets 13: 945-956. http://dx.doi.org/10.1517/14728220903039698

Chahine N, Makhlouf H, Duca L, Martiny L, et al. (2014). Cardioprotective effect of saffron extracts against acute doxorubicin toxicity in isolated rabbit hearts submitted to ischemia-reperfusion injury. Z. Naturforsch., C J. Biosci. 69: 459-470.

Costa LG, Tait L, de Laat R, Dao K, et al. (2013). Modulation of paraoxonase 2 (PON2) in mouse brain by the polyphenol quercetin: a mechanism of neuroprotection? Neurochem. Res. 38: 1809-1818. http://dx.doi.org/10.1007/s11064-013$\underline{1085-1}$

Devaux Y, Vausort M, Azuaje F, Vaillant M, et al. (2012). Low levels of vascular endothelial growth factor B predict left ventricular remodeling after acute myocardial infarction. J. Card. Fail. 18: 330-337. http://dx.doi.org/10.1016/j.cardfail.2012.01.010

$\mathrm{He} \mathrm{H,} \mathrm{Xu} \mathrm{J,} \mathrm{Xu} \mathrm{Y,} \mathrm{Zhang} \mathrm{C,} \mathrm{et} \mathrm{al.} \mathrm{(2012).} \mathrm{Cardioprotective} \mathrm{effects} \mathrm{of} \mathrm{saponins} \mathrm{from} \mathrm{Panax} \mathrm{japonicus} \mathrm{on} \mathrm{acute} \mathrm{myocardial}$ ischemia against oxidative stress-triggered damage and cardiac cell death in rats. J. Ethnopharmacol. 140: 73-82. http:/l dx.doi.org/10.1016/j.jep.2011.12.024

Jeong H, Yim HW, Cho Y, Park HJ, et al. (2013). The effect of rigorous study design in the research of autologous bone marrow-derived mononuclear cell transfer in patients with acute myocardial infarction. Stem Cell Res. Ther. 4: 82. http:/l $\underline{\text { dx.doi.org/10.1186/scrt233 }}$

Jutla SK, Yuyun MF, Quinn PA and Ng LL (2014). Plasma cortisol and prognosis of patients with acute myocardial infarction. J. Cardiovasc. Med. 15: 33-41. http://dx.doi.org/10.2459/JCM.0b013e328364100b

Kivrak T, Sunbul M, Durmus E, Dervisova R, et al. (2014). Acute myocardial infarction due to liquid nicotine in a young man. Ther. Adv. Cardiovasc. Dis. 8: 32-34. http://dx.doi.org/10.1177/1753944713515765

Kong SS, Liu JJ, Hwang TC, Yu XJ, et al. (2011). Tumour necrosis factor- $\alpha$ and its receptors in the beneficial effects of vagal stimulation after myocardial infarction in rats. Clin. Exp. Pharmacol. Physiol. 38: 300-306. http://dx.doi.org/10.1111/ j.1440-1681.2011.05505.x

Lee JH, Park JH, Cho HS, Joo SW, et al. (2013). Anti-biofilm activities of quercetin and tannic acid against Staphylococcus aureus. Biofouling 29: 491-499. http://dx.doi.org/10.1080/08927014.2013.788692

Misra MK, Sarwat M, Bhakuni P, Tuteja R, et al. (2009). Oxidative stress and ischemic myocardial syndromes. Med. Sci. Monit. 15: RA209-RA219.

Rahim MA, Rahim ZH, Ahmad WA and Hashim OH (2015). Can saliva proteins be used to predict the onset of acute myocardial infarction among high-risk patients? Int. J. Med. Sci. 12: 329-335. http://dx.doi.org/10.7150/ijms.11280

Su Y, Wang L and Zhang MZ (2012). Research progress in epidemiology of acute myocardial infarction. Chin. J. Integr. Med. Cardio. Cerebrovasc. Dis. 10: 467-468.

Toldo S, Mezzaroma E, Van Tassell BW, Farkas D, et al. (2013). Interleukin-1 $\beta$ blockade improves cardiac remodelling after myocardial infarction without interrupting the inflammasome in the mouse. Exp. Physiol. 98: 734-745. http://dx.doi. org/10.1113/expphysiol.2012.069831

Yang N, Feng JP, Chen G, Kou L, et al. (2014). Variability in lipid profile among patients presented with acute myocardial infarction, unstable angina and stable angina pectoris. Eur. Rev. Med. Pharmacol. Sci. 18: 3761-3766.

Yao F, Hu ZY, Yan XH, Han H, et al. (2011). Effect of quercetin on the astrocytes gene expression of hypoxia ischemia injury. Chin. J. Neuromed. 10: 1197-1201.

Youn H, Jeong JC, Jeong YS, Kim EJ, et al. (2013). Quercetin potentiates apoptosis by inhibiting nuclear factor-kappaB signaling in H460 lung cancer cells. Biol. Pharm. Bull. 36: 944-951. http://dx.doi.org/10.1248/bpb.b12-01004

Zhang XF, Hu DY, Ding RJ, Wang HC, et al. (2012). Status and trend of cardio-cerebral-vascular diseases mortality in China: data from national disease surveillance system between 2004 and 2008. Zhonghua. Xin Xue Guan Bing Za Zhi 40: 179187.

Zhang Y, Wang J, Guo LL and Wu GJ (2013). Huoxue anxin recipe alleviated peroxidation damage of acute myocardial infarction rats by regulating iNOS/eNOS imbalance: an experimental research. Zhongguo Zhong Xi Yi Jie He Za Zhi 33: 1356-1360.

Zhou L, Sung RY, Li K, Pong NH, et al. (2011). Cardioprotective effect of dexrazoxane in a rat model of myocardial infarction: anti-apoptosis and promoting angiogenesis. Int. J. Cardiol. 152: 196-201. http://dx.doi.org/10.1016/j.ijcard.2010.07.015 